\title{
NICHE: Air-Cherenkov observation at the TA site
}

\author{
Douglas R. Bergman ${ }^{1, *}$, Yoshiki Tsunesada ${ }^{2}$, John F. Krizmanic ${ }^{3}$, and Yugo Omura ${ }^{2}$ \\ ${ }^{1}$ University of Utah, Institute for High Energy Astrophysics \\ ${ }^{2}$ Osaka City University \\ ${ }^{3}$ University of Maryland, Baltimore County, CRESST, NASA Goddard Space Flight Center
}

\begin{abstract}
The Non-Imaging CHErenkov (NICHE) Array has been deployed at the Telescope Array Middle Drum site, and has been collecting data. We see many coincidences with TALE fluorescence mirror events, establishing hybrid imaging/non-imaging air-Cherenkov observation of cosmic ray air showers. We have verified the TALE Profile-Constrained Geometry Fit of Cherenkov events at the $3^{\circ}$ level. We have performed hybrid timing fits between NICHE and TALE and have established that the growth in the FWHM of the NICHE signal with the distance of the shower core depends on the height of the shower maximum.
\end{abstract}

\section{Introduction}

The motivating idea behind the Non-Imaging CHErenkov (NICHE) Array is to use non-imaging light collectors with fine time resolution to sample the air-Cherenkov light pool from cosmic-ray induced air showers. Using the NICHE Array we plan to extend the range of energies observed by TA and TALE to below $1 \mathrm{PeV}$. The energy range of NICHE will overlap with the lower end of the energy range of TALE in air-Cherenkov observations allowing for hybrid imaging/non-imaging observations of air showers. A 14 counter array with 100-m spacing has been deployed in the field-of-view of the TALE telescopes at distances from $600-1000 \mathrm{~m}$. This array was funded by a 18.8M JPY Kakenhi Grant for Young Scientists awarded to Yoshiki Tsunesada. 10 counters were deployed in Sept. 2017, and the remaining 4 couters were deployed in Sept. 2018.

Traditionally, one measures the energy and depth of shower max in non-imaging detector arrays by measuring the shape of the Cherenkov Lateral Distribution (CLD), the flux of Cherenkov radiation from the shower as a function of the distance from the shower axis. Early in the shower, when the average electron energy is high and the transverse momentum is low, the resulting lateral distribution of Cherenkov light at the ground has a peak at about $120 \mathrm{~m}$ from the shower axis due to the typical Cherenkov angle in air of about $1^{\circ}$. As the shower develops and moves lower in the atmosphere the ring gets smeared out as the electrons are of lower energy and less collimated along the shower axis. The smearing mostly fills in the region inside the ring, with the region outside the ring have the same power-law relation as earlier in the shower. Late in the shower development, as the shower near the ground, the center part of the ring is filled in, as the outer parts of the footprint are now at large angles from the shower axis. The result of considering the whole shower is that

*e-mail: bergman@physics.utah.edu the Cherenkov flux at distances above $120 \mathrm{~m}$ from the shower core is proportional to the energy of the shower and has a characteristic power-law dependence on the radius. The lateral distribution within $120 \mathrm{~m}$ depends strongly on depth of shower max, being very flat if $X_{\max }$ is very far away from the ground, and becoming more peaked as $X_{\max }$ moves closer to the ground. This is illustrated in [1]. To accurately measure shower composition, one must sample the Cherenkov wavefront several times within $120 \mathrm{~m}$ of where the shower axis hits the ground. This constrains the size of the array.

As an alternative to the CLD measurements, one can measure the FWHM of the Cherenkov time profile in a given counter. If the counter is close to the shower axis, only late portions of the shower contribute a significant signal, and all the photons arrive within about a nanosecond. This usually leads to just measuring the impulse response waveform for the photodetector. For counters farther from the shower core than $120 \mathrm{~m}$, significant signals come from a large portion of the development of the shower, and because of the varying path lengths, the photons arrive in a range of 10 s to 100 s of nanoseconds. Furthermore, the way the time width grows as a function of distance from the shower axis depends on where the shower reaches its maximum value. This is illustrated in [2]. Thus measuring the Cherenkov Width Lateral Distribution (CWLD) allows one to determine the depth of shower max in a way complementary to the CLD, in that one would like to take measurements far from the shower axis rather than close to it.

\section{Counter Design and Deployment}

The NICHE array has been designed around autonomous counter stations using batteries and solar panels as a power supply and communicating with a base computer on a 

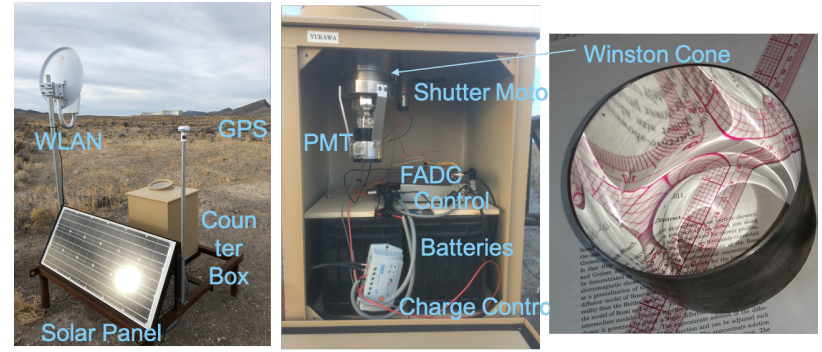

Figure 1. Left: NICHE counter from the outside. Middle: NICHE counter from the inside. Right: A Winston cone for NICHE.

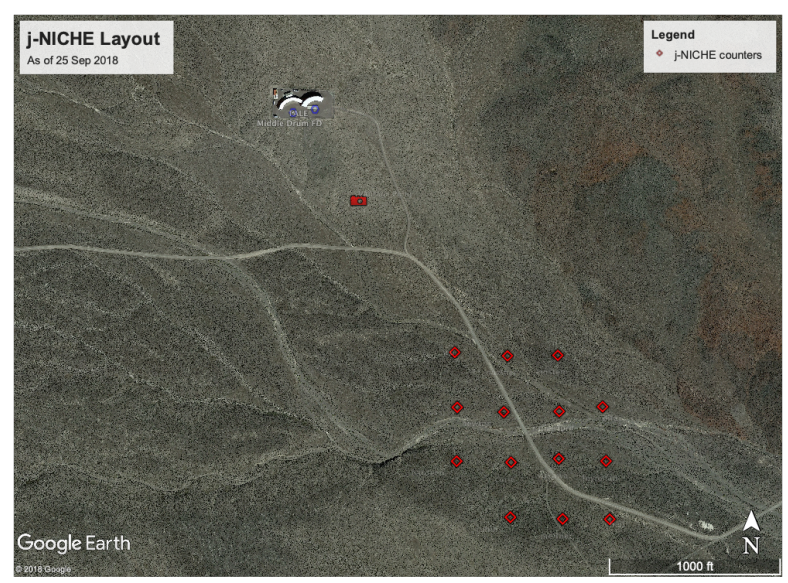

Figure 2. The location of the 14 NICHE counters with respect to the TALE fluorescence site.

WLAN network. Each counter has a GPS antenna to allow coincidences to be determined with better than $15 \mathrm{~ns}$ accuracy. The counter in enclosed in an aluminum box of roughly $50 \times 50 \times 70 \mathrm{~cm}$. The optical detector is a Hamamatsu 3 in. photomultiplier tube. This is connected to a $45^{\circ}$ half-angle acceptance Winston cone. The Winston cone is machined out of a 4 in diameter aluminum dowel and polished to give a good specular reflective surface. The signal waveforms are collected by a $200 \mathrm{MHz}$ 12-bit FADC DAQ system from Brains, Inc. See Figure 1

Fourteen counters have been deployed on a square grid of $100 \mathrm{~m}$ on a side. 10 counters were deployed by 20 September 2017, the beginning of data collection. Four more counters were deployed in September 2018. See Figure 2 .

\section{Status and Preliminary Analyses}

As of 1 October 2018, a total of 1,350 counter-hours of data have been taken, which includes 936,000 individual counter triggers. 160 hours of data have been taken with 8-10 counters operational from 20 September 2017. 20 hours of data have been taken with 12-14 counters operational in September 2018.

One can compare the the gains and thresholds by looking at the integrated signal size distributions.
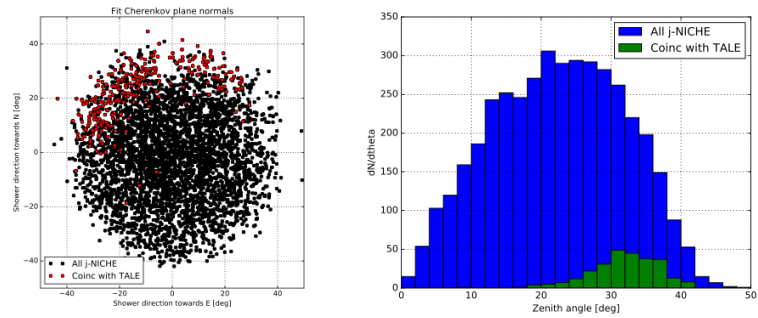

Figure 3. Left: The arrival direction of showers reconstructed from a plane fit to the Cherenkov wave front. Those events which are coincident with TALE events are shown in red. Right: The zenith angle distribution of NICHE plane fit events. Those events coincident with TALE are shown in green.
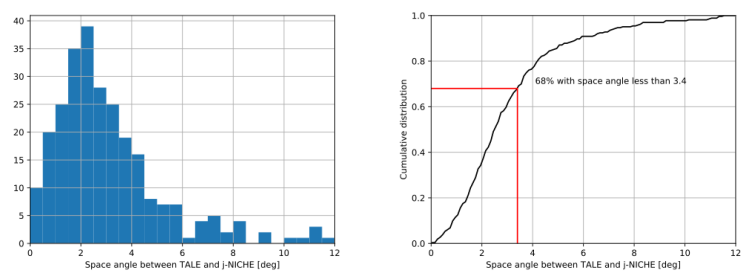

Figure 4. Left: The distribution of the difference in space angle between TALE PCGF fits and NICHE Plane fit. Right: The normalized integral of the space angle distribution.

Using the GPS times stamps, one can look for coincidences between counters. A window of $100 \mu$ s gives essentially no background, given the individual counter trigger rates of about $10 \mathrm{~Hz}$. One can fit the trigger times of coincident counters to the assumption of a plane Cherenkov wavefront. The result of this fit shows a roughly uniform distribution of arrival directions in azimuth, and a zenith angle distribution uniform from $0^{\circ}-$ $30^{\circ}$ and then falling off with nothing beyond $45^{\circ}$. This is what one would expect from the Winston cone acceptance.

We also looked for coincidences between NICHE events and TALE FD events. We found many such coincidences. For coincident events in NICHE, the plane time fit shows the events moving to the NW (towards TALE from NICHE) and predominantly between $25^{\circ}$ and $40^{\circ}$ in zenith angle. This is the expected overlap between the TALE zenith angle acceptance (TALE telescopes point from $30^{\circ}$ to $60^{\circ}$ in elevation and showers must be pointed into the TALE mirrors to be accepted in Cherenkov mode) and the NICHE zenith angle acceptance. See Figure 3.

If we require separate triggers between TALE and a 4 or more NICHE counters, we can compare the direction of showers given by the Profile Constrained Geometry Fit in TALE and the plane fit in NICHE. We find that $68 \%$ of cases agree within $3.4^{\circ}$. This includes an uncertainty in the NICHE plane fit from the non-plane nature of the Cherenkov front which is of order $1^{\circ}$. The TALE PCGF fit should have an accuracy of better than $1^{\circ}$ but it is at least verified here at a somewhat less precise level. See Figure 4

We have also performed a hybrid timing fit using both TALE and NICHE timings. For this fit, the NICHE 

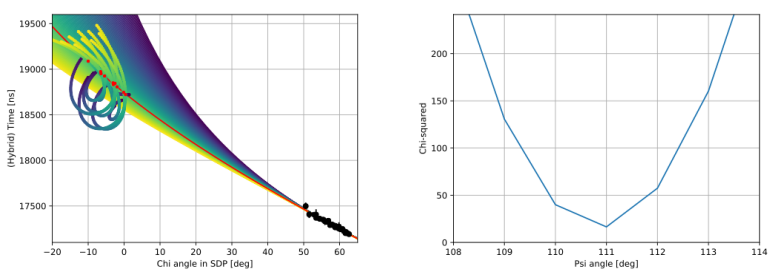

Figure 5. Left: The time vs angle plot of a hybrid time fit. The TALE measurements are shown in black. The various showerdetector-plane angle expectations are shown in green to blue lines. The projection of the NICHE times onto the shower axes are also shown in green to blue points. The best fit time-vsangle line and NICHE points are shown in red. Right: The $\chi^{2}$ distribution of the fit versus the SDP-angle $\psi$.

counter positions are projected onto the shower axis to determine an angle along the shower track for the NICHE counters and the NICHE times are augmented by the time light would take to travel from this point on the shower to the TALE detector. Then a standard monocular timing fit is performed. At first requiring a separate coincidence of 4 or more NICHE counters, we can perform a hybrid fit with one arbitrary extra offset in time between the NICHE counters and the TALE detectors. The offset is then one of the degrees of freedom in the fit. We find in this case that the average offset is $173 \pm 50 \mathrm{~ns}$ and the range of offsets is entirely contained within $50-300 \mathrm{~ns}$. This is very good agreement, given that the TALE waveform sample frequency is $10 \mathrm{MHZ}$.

Fixing the offset at $173 \mathrm{ns,}$ we can also perform hybrid timing fits with all NICHE events, even those with no coincidences within NICHE. We find very good fits in this manner with uncertainties in the sub- $1^{\circ}$ range. See Figure 5 .
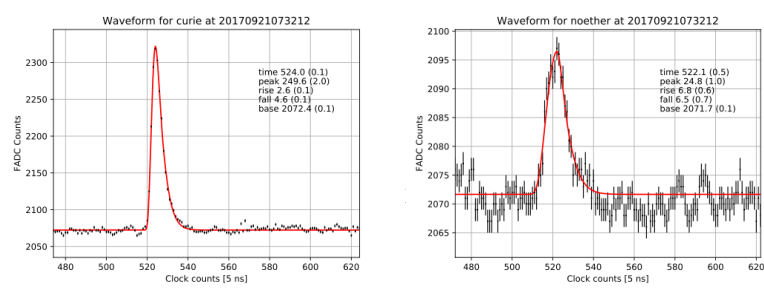

Figure 7. The signal waveforms for two counters in the event from Figure 6: curie and noether.
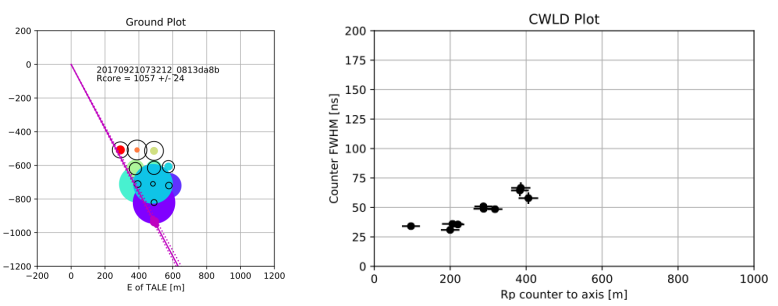

Figure 6. Left: The size, timing and FWHM of NICHE events in a single coincident event. The size of the colored circles shows the size of the signal in the NICHE counter, while the color shows the relative time, from purple to red. The size of the black circle is proportional to the FWHM of the signal. The purple solid and dotted lines show the projection of the TALE shower detector plane onto the ground with errors, and the purple dots show the most likely core position with errors. Right: The FWHM of NICHE signals versus distance from the shower axis for all NICHE counters.

Using TALE PCGF fit to determine where the shower axis hits the ground and how far away the position of shower max is from a given NICHE counter, we can compare the time width of NICHE counters versus the distance from the shower core. Because TALE measures the shower location well above the ground, the exact position of the shower core can be uncertain. We only consider TALE tracks which are $10^{\circ}$ long or longer or which have a duration greater than $400 \mathrm{~ns}$, to reduce the uncertainty in the location of the shower axis at the ground to less than about $100 \mathrm{~m}$. The distance to shower max (from the ground) $D_{\max }$ is in the range of $0-10,000 \mathrm{~m}$. Splitting the $D_{\max }$ distribution up into 10 bins with roughly equal numbers of events, we see that larger $D_{\max }$ corresponds with a wider shower time width at a given distance. See Figure 8.
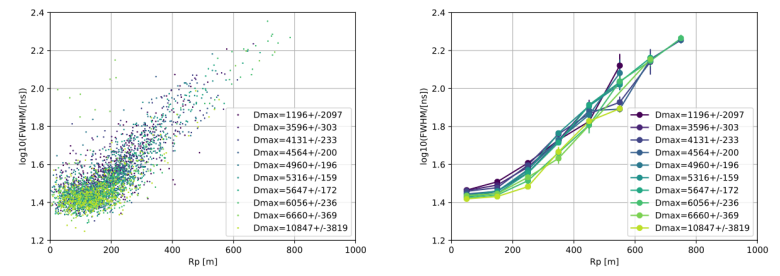

Figure 8. Left: The $\log _{10}$ FWHM vs $R_{P}$ distribution for events with various $D_{\max }$ values. The $D_{\max }$ values in the legend are in meters and the uncertainty represents the width of the $D_{\max }$ bin. Right: A profile plot of the same with the $<\log _{10}$ FWHM $>$ vs shown vs $R_{P}$ distribution for events with various $D_{\max }$ values..

\section{References}

[1] J.R. Patterson, A.M. Hillas, J. Phys. G 9, 1433 (1983)

[2] J.R. Patterson, A.M. Hillas, J. Phys. G 9, 323 (1983) 